\title{
Utility of Prefrontal Space Ratio to Screen for Trisomy 21 in a Racially Diverse Population: A Pilot Study
}

\author{
Barrie Suskin • Anne-Marie Roe $\cdot$ Komal Bajaj
}

Received: 19 May 2014/Revised: 31 May 2014/Accepted: 3 June 2014/Published online: 3 July 2014

(C) Society of Fetal Medicine 2014

\begin{abstract}
To evaluate the prefrontal space (PFS) ratio of fetuses of African descent without Down syndrome and compare it to the PFS ratio previously established in a homogeneous Caucasian population to determine whether there is a difference in the ratio in these two groups. The PFS ratio was calculated retrospectively from stored 2D images of 100 African, African-American and AfricanCaribbean fetuses in the second and third trimester performed at the authors' center. Other data including the maternal age, gestational age, and ethnicity were collected. An unpaired $T$ test was used to compare the previously published Caucasian mean, 0.97 (SD 0.29) with the mean established for the black population 1.37 (SD 0.44). The difference between these two PFS ratios was found to be significant $(p<0.0001)$. This pilot study suggests a significant difference in the PFS in the non-Caucasian population. In order to use the PFS as a sonographic screening tool for Down syndrome, it will be important to determine normal values in different ethnic populations.
\end{abstract}

Keywords Prenatal screening - Aneuploidy · Down syndrome

\section{B. Suskin $(\bowtie)$}

Department of Obstetrics and Gynecology and Women's Health, Montefiore Medical Center, Albert Einstein College of Medicine, 1695 Eastchester Road, Suite 301,

Bronx, NY 10461, USA

e-mail: Bsuskin@montefiore.org

A.-M. Roe · K. Bajaj

Department of Obstetrics and Gynecology, North Bronx Health Network, New York, NY, USA

\section{Introduction}

Trisomy 21 (Down syndrome) is the most common chromosomal abnormality in live born infants [1,2]. Over the years, there have been great strides in the various strategies for prenatal screening for trisomy 21 that utilize maternal serum markers, ultrasound, and most recently, cell-free fetal DNA in the maternal circulation [3-6]. However, routine ultrasounds in the second and third trimester provide another opportunity for screening and the potential to provide patients with more information regarding the genetic risks to their fetuses.

The characteristic facial features of trisomy 21 , including the dorsal displacement of the edge of the maxilla as well as thickening of the prenasal skin, have been well described [1]. The prefrontal space (PFS) ratio capitalizes upon these changes and has been shown to be an effective screening marker for trisomy 21 when calculated from midsagittal 2D sonographic images of the fetal profile in the second and third trimester [7, 8]. However, up to this point, these limited studies have been performed in Caucasian subjects, or subjects where race data was not reported. As facial morphology varies amongst different racial groups, it is plausible that the normal PFS ratio may differ in non-Caucasian fetuses $[9,10]$. The objective of this pilot study was to evaluate the PFS ratio of fetuses of African, AfricanAmerican and African-Caribbean descent without Down syndrome to determine whether there is a difference in the ratio in these two groups. If a true difference exists, it could impact the utility of this ratio as a screening tool for Down syndrome in racially diverse patient populations.

\section{Materials and Methods}

After Institutional Review Board approval, consecutive birth records from October to December 2012, from the 


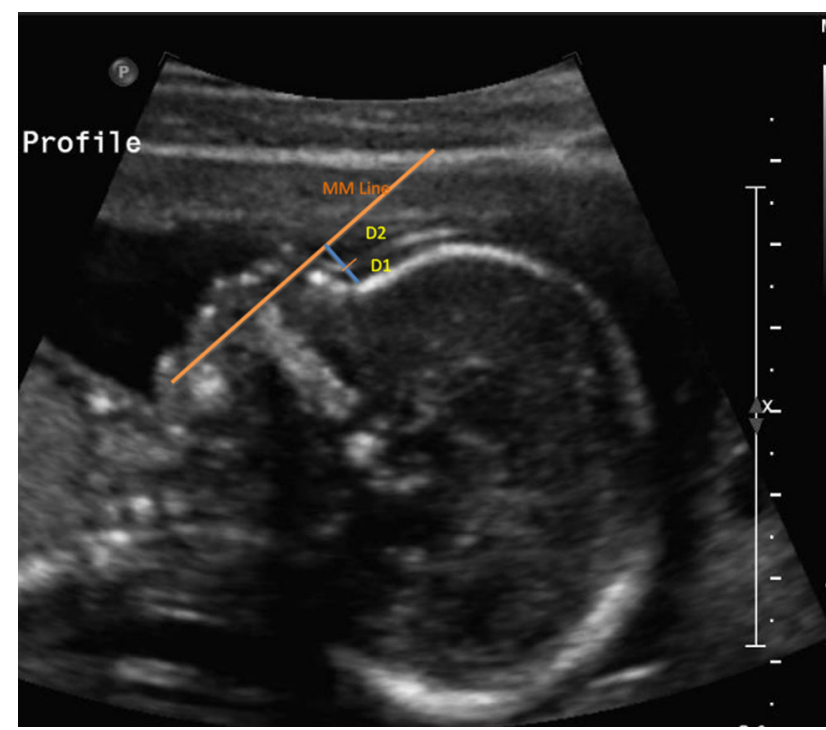

Fig. 1 Calculating the PFS ratio. First the mandibula-maxilla line (MM line), which is an extension of a line from the leading edges of both bones, was drawn. D1 was calculated as the distance from the leading edge of the frontal bone to the skin edge. D2 was then drawn from the skin edge to the MM line. The PFS was then calculated by dividing $\mathrm{D} 2$ by $\mathrm{D} 1$

authors' large urban hospital were utilized to identify subjects who self-identified as "black". Subjects were excluded if they carried multiple gestations; if the fetus had a known genetic aberration or was identified to have any phenotypic abnormalities, including any features associated with Down syndrome, in the newborn period. The PFS ratio was retrospectively calculated from stored $2 \mathrm{D}$ images of the African, African-American and non-Hispanic African-Caribbean fetuses in the second and third trimester. These prenatal sonograms were performed in a large outpatient maternal-fetal testing unit under the strict supervision of reproductive geneticists and maternal-fetal medicine specialists. The images were excluded if the fetal profile was not truly midsagittal or if the anterior edges of the maxilla and skin were not clearly identifiable. The PFS ratio (as diagramed in Fig. 1) was measured by first creating the mandibula-maxilla line (MM line) which is an extension of a line from the leading edges of both bones. D1 was calculated as the distance from the leading edge of the frontal bone to the skin edge. D2 was then drawn from the skin edge to the MM line. The PFS was then calculated by dividing D2 by D1. Controls were performed in a sample of Caucasian patients to demonstrate the consistency of the present methods with the previously established mean from Yazdi et al. [7].

An unpaired $T$ test was used to compare the PFS ratio in the Caucasian group previously established in Yazdi et al. [7], with the value in the self-identified black population.
Table 1 Characteristics of maternal subjects and their pregnancies

\begin{tabular}{llc}
\hline $\begin{array}{l}\text { Euploid population } \\
\text { characteristics }\end{array}$ & $\begin{array}{l}\text { Yazdi et al. [7] } \\
\mathrm{n}=279\end{array}$ & $\begin{array}{l}\text { Suskin et al. } \\
\mathrm{n}=100\end{array}$ \\
\hline $\begin{array}{l}\text { Maternal age in y, median } \\
\text { (range) }\end{array}$ & $30.5(19.0-45.9)$ & $27.8(15-54)$ \\
$\begin{array}{l}\text { Gestational age in wk, median } \\
\text { (range) }\end{array}$ & $21.1(15.0-40.0)$ & $23.4(18.0-37.4)$ \\
$\begin{array}{l}\text { Second trimester examinations, } \\
\mathrm{n}(\%)\end{array}$ & $208(75.4 \%)$ & $71(71 \%)$ \\
$\begin{array}{c}\text { Third trimester examinations, } \\
\mathrm{n}(\%)\end{array}$ & $68(24.6 \%)$ & $19(19 \%)$ \\
$\begin{array}{l}\text { Caucasian, n }(\%) \\
\text { Self-identified blacks, n (\%) }\end{array}$ & $279(100 \%)$ & $0(0 \%)$ \\
\hline
\end{tabular}

Table 2 PFS ratio in normal fetuses

\begin{tabular}{lllll}
\hline Study & N & $\begin{array}{l}\text { D1 frontal } \\
\text { os-skin }\end{array}$ & $\begin{array}{l}\text { D2 skin- } \\
\text { MM line }\end{array}$ & $\begin{array}{l}\text { Prefrontal space } \\
\text { ratio (D2/D1) } \\
\text { mean (SD) }\end{array}$ \\
\hline Yazdi et al. [7] & 279 & $4.6(1.4)$ & $4.4(1.6)$ & $0.97(0.29)$ \\
Suskin Kaplan et al. & 100 & $4.9(2.0)$ & $6.6(3.1)$ & $1.37(0.44)$ \\
\hline
\end{tabular}

Data including maternal age, gestational age, and ethnicity was also collected (Table 1).

\section{Results}

The patients with singleton deliveries, self-identified as black were 162. These charts were reviewed and 62 were excluded. Forty-one were excluded based upon the lack of prenatal care or Level II imaging in the system, 17 due to suboptimal images, and 2 due to the presence of fetal abnormalities. The PFS was calculated as above for the remaining 100 fetuses (Table 2).

The Caucasian population was found to have a mean of 1.01 (SD 0.27), very similar to Yazdi et al. [7]. An unpaired $T$ test was used to compare the previously published Caucasian mean, 0.97 (SD 0.29) from Yazdi et al., with the mean established for the black population 1.37 (SD 0.44). The difference of euploid PFS ratio was found to be significant with a $p<0.0001$.

\section{Discussion}

PFS ratio has the potential to be an excellent sonographic screening tool for Down syndrome for multiple reasons including relatively low cost, reasonable accessibility and timing of the testing. Unlike other screening tests, it does not require additional ultrasounds, clinic visits or studies on 
blood [11, 12]. Additionally, although there are multiple advantages of early diagnosis, there are many patients who present too late for the screening that is performed earlier in pregnancy, whereas the PFS can be done at any point in time after 15 weeks gestation. By utilizing the inherent differences in facial morphologies of euploid versus fetuses with Down syndrome, providers would be able to give their patients important additional information about their pregnancy.

Consistency of the present methods was confirmed by comparing the mean values in Caucasians in the present study with that of Yazdi et al. [7]. Due to the fact that the total number calculated for the Caucasians was greater in the latter study, the decision was made to use that as the mean for the calculations.

Although the authors found a significant difference in their population as compared with a Caucasian population, the study population had large variance in its results. This could be due to multiple factors. First, the only mechanism of determining patient's race was based upon the latter's self-reported identity. In addition, as in the pre-existing studies describing PFS, paternal race was not assessed. Also, there could be significant intraracial differences in facial morphology. These may pose limitations if attempts to make this type of testing were widespread.

\section{Conclusions}

The PFS for the described black population in the present study was significantly different from a homogeneous Caucasian PFS. In light of this pilot study, PFS ratios may have a normal variation influenced by the variation in facial morphology. This suggests that knowing a patient's racial group could be quite important in determining the utility of the PFS ratio. It will be imperative to further investigate the mean values in euploid, in larger number of fetuses and in other racial groups, before using the PFS ratio as a tool for Down syndrome screening.

Conflict of interest None.

\section{References}

1. Jones KL. Down syndrome. Smith's recognizable patterns of human malformation. 6th ed. Philadelphia: Elsevier Saunders; 2006. p. 7.

2. Epstein CJ. Down syndrome (trisomy 21). In: Scriver CR, Beaudet AL, Sly WS, Valle D, editors. The metabolic and molecular bases of inherited disease. 8th ed. New York: McGraw-Hill; 2001. p. 1223.

3. Driscoll D, Gross S. Prenatal screening for aneuploidy. N Engl J Med. 2009;360:2556-62.

4. Alldred SK, Deeks JJ, Guo B, et al. Second trimester serum tests for Down's syndrome screening. Cochrane Database Syst Rev. 2012;6:CD009925.

5. ACOG Committee on Practice Bulletins. ACOG Practice Bulletin No. 77: screening for fetal chromosomal abnormalities. Obstet Gynecol. 2007;109:217.

6. ACOG Committee on Practice Bulletins. ACOG Practice Bulletin No. 545: noninvasive prenatal testing for fetal aneuploidy. Obstet Gynecol. 2012;120:1532-4.

7. Yazdi B, Sonek J, Oettling C, et al. The prefrontal space ratio in second and third trimester screening for trisomy 21 . Ultrasound Obstet Gynecol. 2013;41:262-6.

8. Sonek J, Molina F, Nicolaides KH, et al. Prefrontal space ratio: comparison between trisomy 21 and euploid fetuses in the second trimester. Ultrasound Obstet Gynecol. 2012;40(3):293-6.

9. Farkas L, Katic M, Forrest C. International anthropometric study of facial morphology in various ethnic groups/races. J Craniofac Surg. 2005;16(4):615-46.

10. Vioarsdottir U, O'Higgins P, Stringer C. A geometric morphometric study of regional differences in the ontogeny of the modern human facial skeleton. J Anat. 2002;201(3):211-29.

11. Odibo AO, Stamilio DM, Nelson DB, et al. A cost-effectiveness analysis of prenatal screening strategies for Down syndrome. Obstet Gynecol. 2005;106:562-8.

12. Harris RA, Washington AE, Nease RF Jr, Kuppermann M. Cost utility of prenatal diagnosis and the risk-based threshold. Lancet. 2004;363:276-82. 